\title{
Supplementary information: \\ A generalizable single-chip calibration method for highly quantitative SERS via Inkjet Dispense
}

Fausto D’Apuzzo*, Raghuvir N. Sengupta, Milo Overbay, Jason S. Aronoff, Anita Rogacs and Steven J. Barcelo

HP Inc. Laboratories, 1501 Page Mill Road, Palo Alto, CA

* corresponding author: fausto.dapuzzo@hp.com

Table of Content:

\section{Contents}

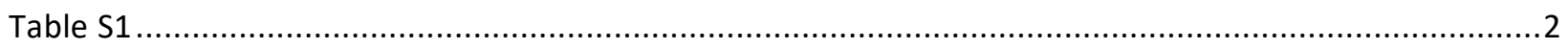

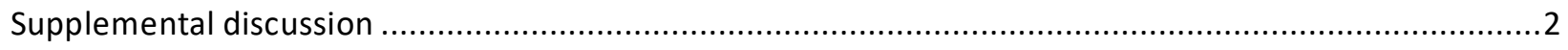

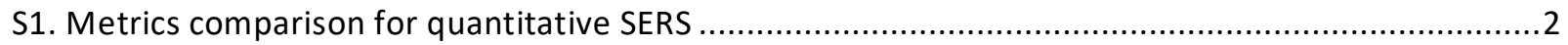

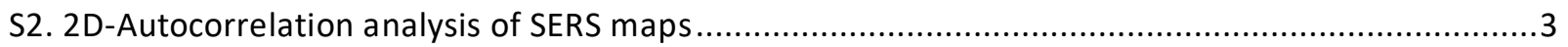

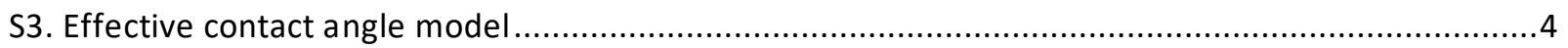

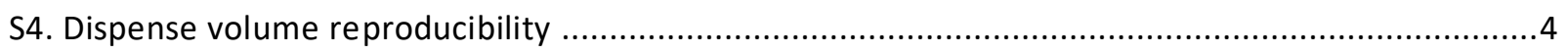

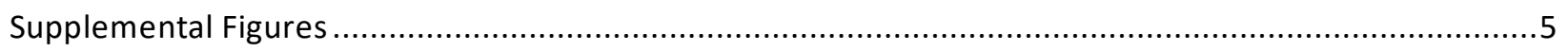

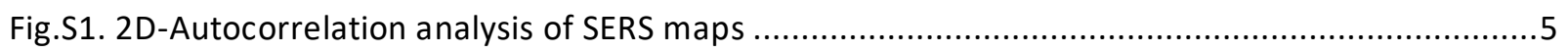

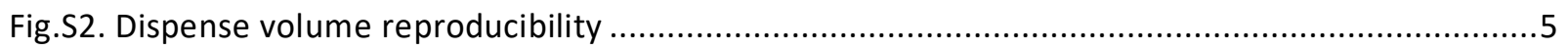

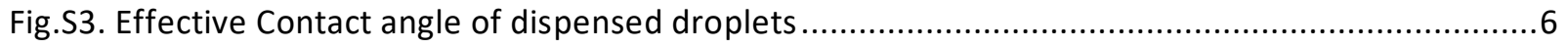

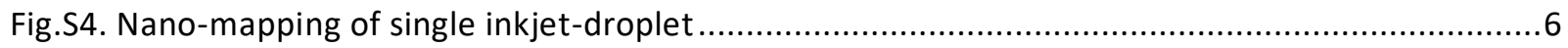

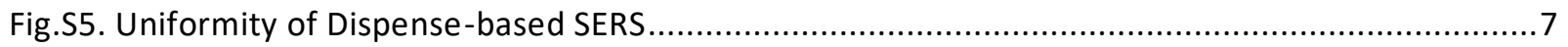

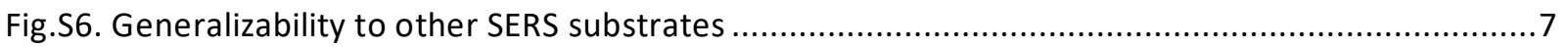

Fig.S7. Quantitation and dynamic range for Adenine ................................................................ 8

Fig.S8. Quantitation and dynamic range for Pyocyanin .......................................................... 8

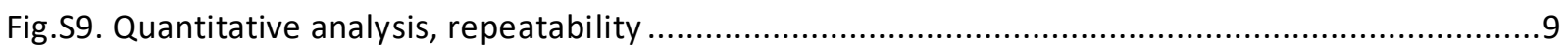

Fig.S10. Effect of firing frequency and volume on drying time: .................................................

Appendix S1

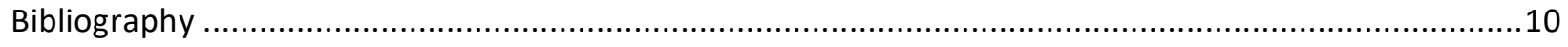




\section{Table S1}

S1. Literature comparison of relevant quantitative SERS data from a literature survey. The $R^{2}$ and RSE are either as reported or calculated from the Figure of Merit (FOM) of quantitation performance as reported in the publication. The definition of different FOMs is reported in the supplementary section S1. (N.R. = Non-Reported)

\begin{tabular}{|c|c|c|c|c|c|c|c|c|c|}
\hline Authors & Ref. & Year & Method & Incub. time & LOD & Substrate CV (\%) & $R^{2}$ & RSE (\%) & FOM \\
\hline This work & & 2019 & in situ calibration & $200 \mathrm{~ms}$ & $30 \mathrm{nM}$ & 10 & 0.9997 & 1.7 & RME \\
\hline J. U. Lee et al. & [1] & 2019 & Calibration & $12 \mathrm{~h}$ & $100 \mathrm{aM}$ & 2.89 & 0.973 & 16.4 & RSD \\
\hline X. Wang et al. & {$[2]$} & 2018 & Calibration & $1 \mathrm{~h}$ & $1 \mathrm{nM}$ & N.R. & 0.98 & 14.1 & $\mathrm{R}^{2}$ \\
\hline S. Zou et al. & [3] & 2018 & Standard addition & $30 \mathrm{~m}$ & $10 \mathrm{nM}$ & N.R. & 0.996 & 6.3 & $\mathrm{RE}$ \\
\hline X-Q. Zhang et al. & [4] & 2018 & Internal standard & $15 \mathrm{~m}$ & $50 \mathrm{nM}$ & N.R. & 0.994 & 8.0 & ARD \\
\hline K. Wu et al. & [5] & 2017 & Calibration & $5 \mathrm{~s}$ & $300 \mathrm{nM}$ & 7 & N.R. & N.R. & RSD \\
\hline C-X. Shi et al. & [6] & 2017 & Internal standard & N.R. & $40 \mathrm{nM}$ & N.R. & 0.997 & 5.8 & $\mathrm{RE}$ \\
\hline J. Guicheteau et al. & [7] & 2017 & Calibration & $20 \mathrm{~m}$ & $5 \mathrm{nM}$ & N.R. & 0.97 & 17.3 & $\mathrm{R}^{2}$ \\
\hline Y. Tian et al. & {$[8]$} & 2016 & Calibration & $5 \mathrm{~m}$ & $50 \mathrm{nM}$ & N.R. & 0.988 & 11.0 & $\mathrm{R}^{2}$ \\
\hline C. Zhu et al. & [9] & 2016 & Calibration & $3 \mathrm{~h}$ & $10 \mathrm{nM}$ & 7.2 & 0.974 & 16.1 & $\mathrm{R}^{2}$ \\
\hline Y. Zou et al. & {$[10]$} & 2016 & Internal standard & N.R. & $\sim p M$ & 7 & 0.994 & 7.7 & $\mathrm{R}^{2}$ \\
\hline Y. Wang et al. & [11] & 2016 & Isotope probing & N.R. & N.A. & N.R. & 0.9982 & 4.2 & $\mathrm{R}^{2}$ \\
\hline W. Shen et al. & [12] & 2015 & Internal standard & $10 \mathrm{~m}$ & $0.1 \mathrm{pM}$ & N.A. & N.R. & N.R. & N.R. \\
\hline Y. Chen et al. & {$[13]$} & 2015 & Internal standard & $2 \mathrm{~m}$ & $90 \mathrm{nM}$ & N.R. & 0.998 & 4.8 & ARPE \\
\hline A. Fales et al. & [14] & 2015 & Internal Standard & N.R. & $1 \mathrm{uM}$ & N.R. & 0.9946 & 7.3 & $\mathrm{R}^{2}$ \\
\hline J. Song et al. & [15] & 2014 & Internal standard & $2 \mathrm{~m}$ & $100 \mathrm{nM}$ & N.R. & 0.999 & 3.2 & ARPE \\
\hline T. Xia et al. & [16] & 2014 & Internal standard & N.R. & $60 \mathrm{nM}$ & N.R. & 0.996 & 6.0 & ARPE \\
\hline J. Yang et al. & {$[17]$} & 2013 & Calibration & $1 \mathrm{~h}$ & $\mathrm{pM}$ & N.R. & 0.98 & 14.1 & $\mathrm{R}^{2}$ \\
\hline X. Jian et al. & [18] & 2013 & Calibration & $3 \mathrm{~h}$ & $200 \mathrm{nM}$ & 5.8 & 0.97 & 17.3 & $\mathrm{R}^{2}$ \\
\hline D-K. Lim et al. & [19] & 2011 & Internal Standard & $12 \mathrm{~h}$ & $2 \mathrm{pM}$ & N.R. & 0.986 & 11.8 & $\mathrm{R}^{2}$ \\
\hline A. Marz et al. & {$[20]$} & 2009 & Isotopologue & $30 \mathrm{~s}$ & $1 \mathrm{mM}$ & N.R. & N.R. & N.R. & N.R. \\
\hline M. Sackmann et al. & [21] & 2006 & Calibration & $30 \mathrm{~m}$ & $100 \mathrm{nM}$ & N.R. & 0.99 & 10.0 & $\mathrm{R}^{2}$ \\
\hline
\end{tabular}

\section{Supplemental discussion}

\section{S1. Metrics comparison for quantitative SERS}

Quantitative performance in the SERS literature review (Supplementary Table S1) is reported with the following Figure of Merits (FOM):

\section{- Relative Measurement Error (RME)}

- Relative Error (RE)

- Average Relative Deviation (ARD)

- Average Relative Prediction Error (ARPE)

- Relative Standard Deviation (RSD)

For the sake of simplicity and comparison, we considered the Relative Standard Error (RSE) which we report in percentage. When the RSE is not directly reported, it can be calculated from the $R^{2}$ value using the relation: 


$$
\operatorname{RSE}(\%)=100 \cdot \sqrt{1-R^{2}}
$$

This relation relies on the fact that the RSE, as defined, is related to the Fraction of Variance Unexplained (FVU). In fact, the $\mathrm{R}^{2}$ represents the fraction of variance explained by a model (linear or not), while the FVU is its quadratic complement (i.e. FVU $=1-\mathrm{R}^{2}$ ). Furthermore, $\mathrm{RSE}=100 \cdot \sqrt{F V U}$ represents the standard error "not explained" by the model and it is a direct quantification of the predictive confidence of a measurement using the model.

\section{S2. 2D-Autocorrelation analysis of SERS maps}

Autocorrelation analysis has been validated on multiple substrates both from our group as well as on map data published by another group ${ }^{22}$. The map analysis aims at evaluating the coefficient of variation (CV) of the intensity as a function of correlation distance $d$. We start with the standard definition of CV:

$$
C V \equiv \frac{\text { standard deviation }}{\text { mean }}=\frac{\sqrt{\frac{\sum_{x} \sum_{y}\left(I_{x, y}-<I>\right)^{2}}{N^{2}}}}{<I>}
$$

where $\langle I\rangle$ is the mean value across the intensity map $I_{x, y}, x$ and $y$ are the row and column indexes, and $N^{2}$ is the total number of pixels in a 2D square array map (of side $N$ ). We no notice that the CV can be rewritten as:

$$
\begin{aligned}
C V & =\sqrt{\frac{\left.\sum_{x} \sum_{y}\left(I^{2}+<I\right\rangle^{2}-2 I<I>\right)}{\left.N^{2}<I\right\rangle^{2}}} \\
& =\sqrt{\frac{\left\langle I^{2}\right\rangle}{\langle I\rangle^{2}}-1}
\end{aligned}
$$

We can now consider the definition of the normalized 2D-autocorrelation $G_{\delta_{x}, \delta_{y}}$ as a function of x-and $y$ - correlation distance ( $\delta_{x}$ and $\delta_{y}$ respectively), in the discrete case:

$$
G_{\delta_{x}, \delta_{y}} \equiv \frac{1}{N^{2}<I^{2}>} \sum_{x, y}\left|I_{x+\delta_{x}, y} I_{x, y+\delta_{y}}\right|
$$

Where this expression is normalized to 1 for $\delta_{x}=0$ and $\delta_{y}=0$, so that:

$$
G_{0,0}=\frac{1}{N^{2}<I^{2}>} \sum_{x, y}\left|I_{x, y}^{2}\right|=1
$$

Here for simplicity we also define the axis-averaged 2D autocorrelation as $G_{\delta}=\frac{1}{2}\left(G_{\delta_{x}, 0}+G_{0, \delta_{y}}\right)$ for the isotropic case. Finally, we compute the average value of $G_{\delta}$, which yields (see Appendix 1 ):

$$
<G_{\delta}>=\frac{<I>^{2}}{<I^{2}>}
$$

We can now re-write the definition of $C V$ in relation to $G(\delta)$ as:

$$
C V=\sqrt{\frac{1}{\left.<G_{\delta}\right\rangle}-1}
$$

Page | S-3 
where $\left\langle G_{\delta}>\right.$ denotes the average over the different correlation distances. At this point it intuitive to define $C V_{\delta}$ as an extension of the previous relation:

$$
C V_{\delta} \equiv \sqrt{\frac{1}{G_{\delta}}-1}
$$

We can validate the physical meaning of this definition by noticing that for $\delta=0$ any signal is perfectly autocorrelated, and therefore $G_{\delta}=1$ and $C V_{\delta}=0$. On the other hand, for $\delta \rightarrow \infty$ there will be some (case-dependent) degradation of the autocorrelation and in the extreme case $G_{\delta} \rightarrow 0$, which would correspond to a $C V_{\delta} \rightarrow \infty$, which intuitively represents a signal that completely lost auto-correlation. We also notice that the above definition of $C V_{\delta}$ reduces to the traditional one for $C V$ when considering the average value of $G_{\delta}$

We also point out that in Fig. 2e of the main text, the trend is only visible for the lowest magnifications $(4 x, 5 x, 20 x)$ and not for the highest magnification (50x). This is a result of variability adding quadrat ically such that $C V^{\text {tot }}=\sqrt{\left(C V^{\text {intr }}\right)^{2}+\left(C V^{\text {spatial }}\right)^{2}}$, where $C V^{\text {intr }}$ is the intrinsic variation due to the magnification chosen. Thus, we see that while the spatial component accounts for about $10 \%$ increase from the micron to the millimeter scale at low magnification, at the highest magnification where the intrinsic $\mathrm{CV}$ is much higher (i.e. $C V_{50 x}^{\text {intr }}=45 \%$ ) the expected quadratic increase would only amount to only $\sim 1 \%$ (i.e. $C V^{t o t} \sim 46 \%$ ).

\section{S3. Effective contact angle model}

We measured the effective contact angle of inkjet droplets (Fig.S3). Given the fast drying behavior of the dispensed droplets, a static contact angle measurement was not possible. For this reason, we inferred the effective contact angle by fitting a static contact angle model to the observed footprint of the dried droplets on the sensor surface as a function of dispensed volume.

For a given contact angle $\vartheta$, and dispensed volume $\mathrm{V}$, the spherical cap model yields a diameter of the cap base given by the relation:

$$
d(V, \vartheta)=2 \times 10^{8} \sin \vartheta \sqrt[3]{\frac{3 V}{\pi(2+\cos \vartheta)(1-\cos \vartheta)^{2}}}
$$

We then experimentally measured $d(V)$ for water and, for comparison, ethanol and fitted the model to the experimental data with the free parameter $\vartheta$. The fitting yields $\vartheta_{\text {water }}=110^{\circ}$ and $\vartheta_{\text {ethanol }}=3^{\circ}$. These values are lower compared to what is expected from a static measurement of contact angle on the same hydrophobic surface (not shown, $\vartheta_{\text {water }}=150^{\circ}$ ) and this difference can be ascribed to the impact momentum ( $10 \mathrm{~m} / \mathrm{s}$ from inkjet dispensing) which facilitates spreading of the droplets, pinning the contact line on a larger footprint than in the static case or from wicking of the fluid through the nanostructures surface.

\section{S4. Dispense volume reproducibility}

We measured the variability of the droplet diameter of single droplets dispensed on a sensor and observed a coefficient of variation $\left(\frac{\sigma_{d}}{d}\right)_{\text {measured }} \sim 2.25 \%$ for the diameter (Fig.S2). The reproducibility 
of droplet volumes $(V)$ from one dispense event to the next is reported in the specs of the HP D300 Digital Dispenser to be less than $8 \%$. From the propagation of uncertainty:

$$
\frac{\sigma_{V}}{V}=3 \cdot \frac{\sigma_{d}}{d}
$$

the relative standard deviation of the diameter due to the Dispenser is expected to be $\left(\frac{\sigma_{d}}{d}\right)$ specifications

$\sim 2.7 \%$, consistent with the measured coefficient of variation.

\section{Supplemental Figures}

Fig.S1. 2D-Autocorrelation analysis of SERS maps
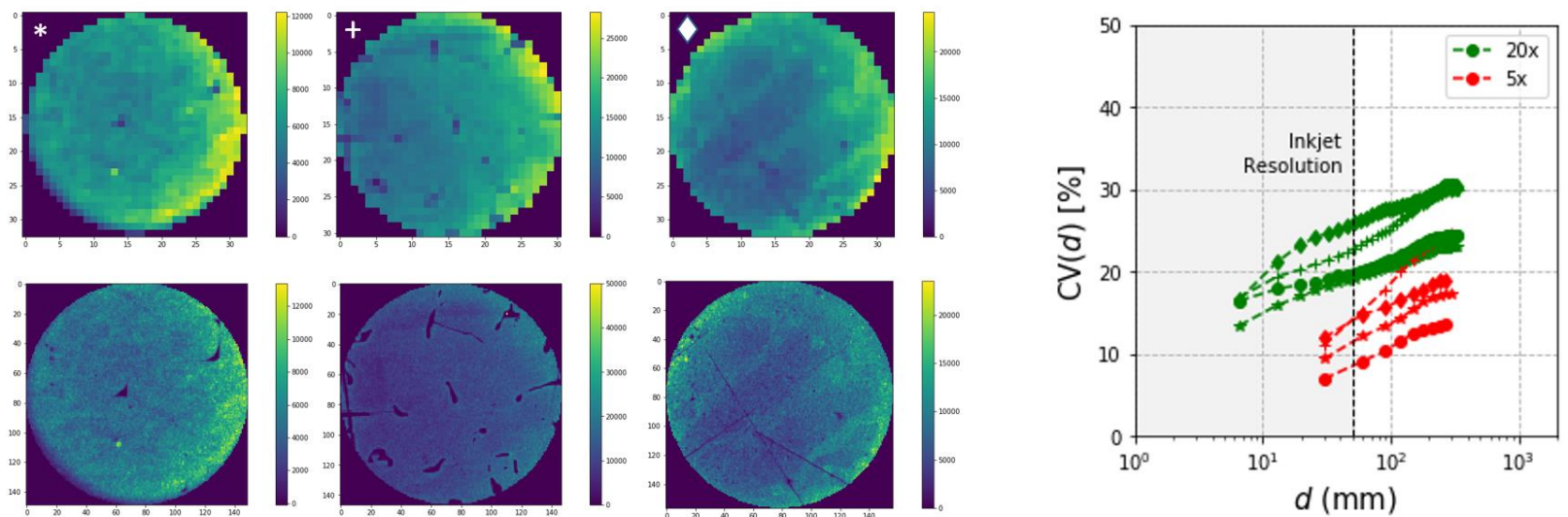

Figure S1. Raman intensity maps and CV(d) for three sensors soaked in aqueous BPE and two magnifications: 5x (upper) and 20x (lower). Corresponding CV(d) for all samples (including data in main text).

Fig.S2. Dispense volume reproducibility

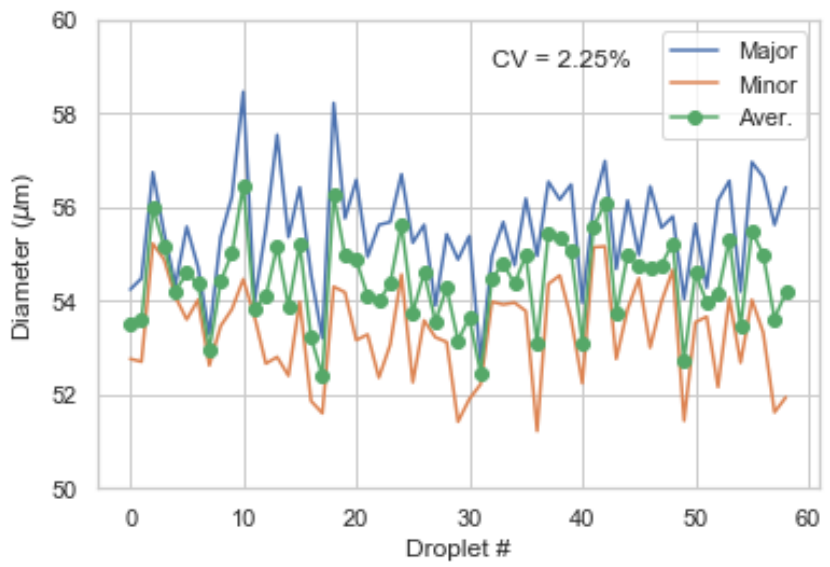

Figure S2. Statistical evaluation of the footprint of dried droplets for the fixed volume (28 $\mathrm{pL}$ ) as measured by fitting an ellipsis to the microscope image (20x), as described in Supplementary Discussion S2. The ellipsis major (blue), minor (orange) and average (green) full axis is reported. Average value is $54.2 \mu \mathrm{m}$, Standard deviation is $1.2 \mu \mathrm{m}$, and $\mathrm{CV}=2.25 \%$. 
Fig.S3. Effective Contact angle of dispensed droplets

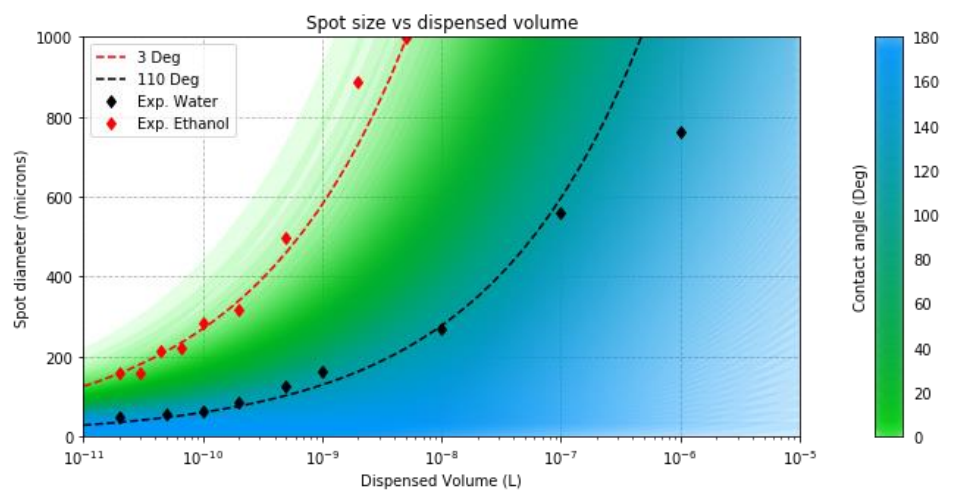

Figure S3. Spot size diameter of the dried droplets as a function of volume for both water (black) and ethanol (red). Fitting of the experimental data to a spherical cap model (dotted lines and color scale) estimates the effective contact angle (color scale) as describe in the Supplementary Discussion S3.

Fig.S4. Nano-mapping of single inkjet-droplet

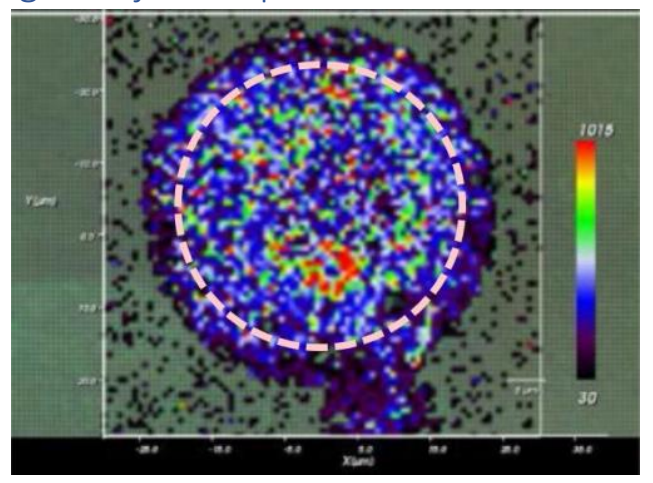

Figure S4. Overlay of microscopy image and SERS intensity nano-map collected with 100X objective stepped with 700nm increments over a single dispensed droplet of BPE (1 $\mu \mathrm{M})$. The $40 \mu \mathrm{m}$ laser beam size (from the $4 x$ objective) is reported (pink) for comparison. 
Fig.S5. Uniformity of Dispense-based SERS

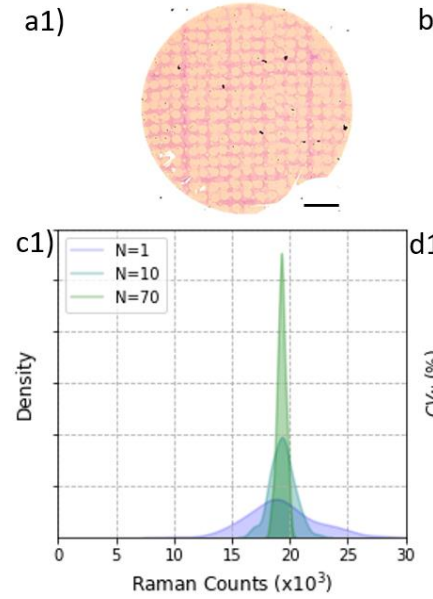

b1)
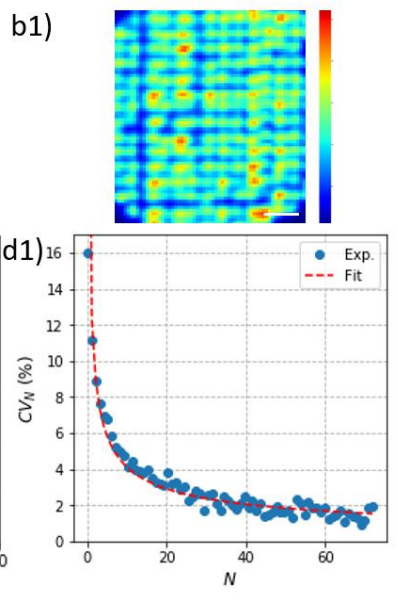

a2)
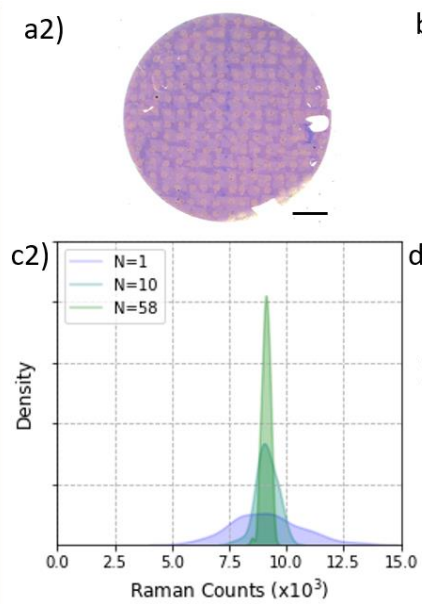

b2)
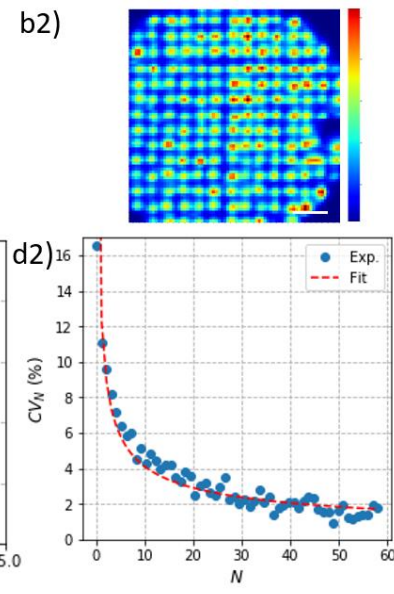

Figure 55. Microscope image (a1, a2), Raman intensity map at $1203 \mathrm{~cm}^{-1}(b 1, b 2)$, histograms of the Raman intensity across all droplets in the maps $(c 1, c 2)$ and for $N=10$ and $N=70$ random subsets. Scaling of $C V_{N}$ with the size $N$ of the random subsets averaged (d1, d2). Scale bars: $200 \mu \mathrm{m}$.

Fig.S6. Generalizability to other SERS substrates

a)

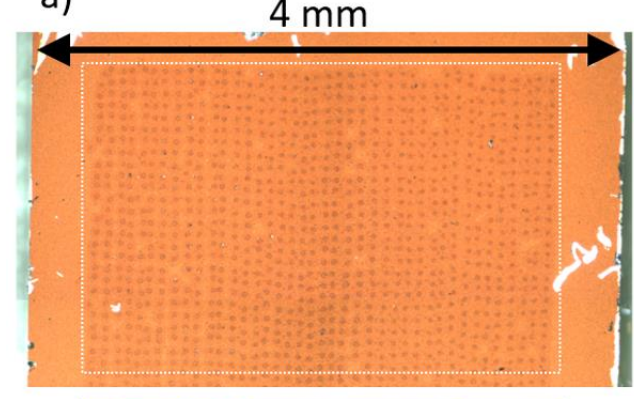

b)

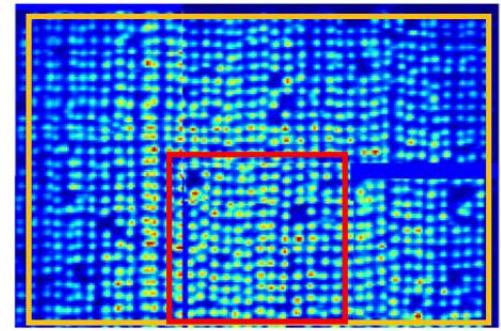

c)

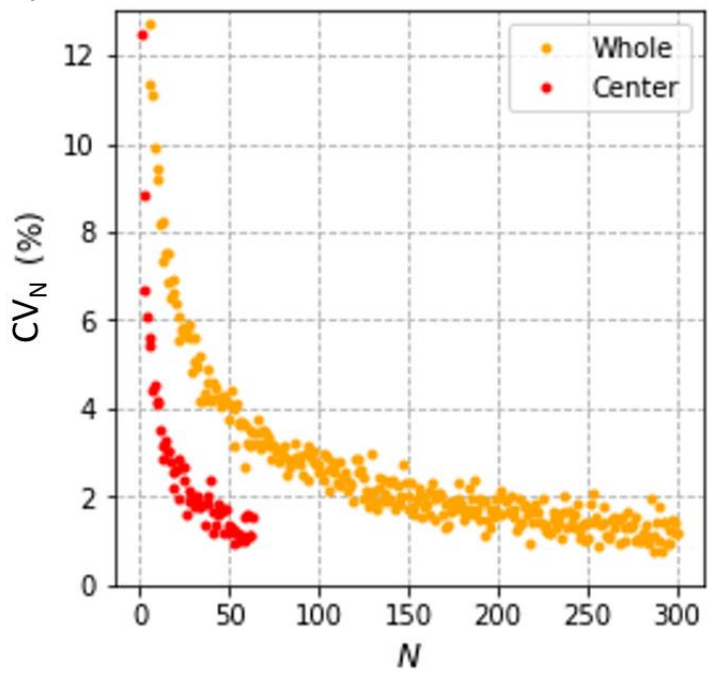

Figure S6. Microscope image of a commercial SERS substrate (Silmeco ApS) spotted with an array of 1000 droplets (a) of aqueous BPE (300 MM). SERS intensity ma at $1203 \mathrm{~cm}^{-1}$ (b). Scaling of $C V_{N}$ of the average SERS intensity of a subset of $N$-droplets randomly chosen across the whole array (orange), or within the most uniform subset in the center (red). 
Fig.S7. Quantitation and dynamic range for Adenine
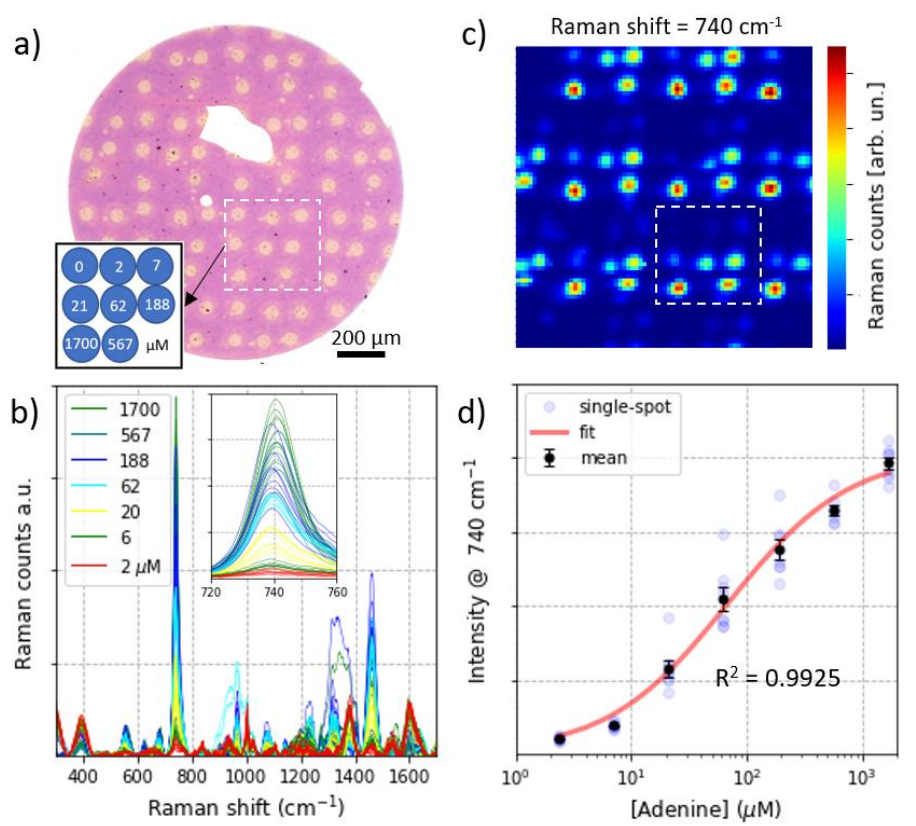

Figure S7. Microscope image of a sensor spotted with eight concentration of adenine (0, 2, 7, 21, 62, 188,567 and $1700 \mu M$ ) (a). SERS intensity map at the $740 \mathrm{~cm}^{-1}$ Raman shift, which has been assigned to the adenine ring breathing mode [23]. (b). Spectra for each droplet in the map color coded by concentration (c). Raw and average intensity as a function of concentration. Fitting the averaged data to the Hill equation yields an $R^{2}$ of 0.9925 , with $K_{d}=3.66 \cdot 10^{-4} \mathrm{M}$ and $n=0.86$.

Fig.S8. Quantitation and dynamic range for Pyocyanin

a)

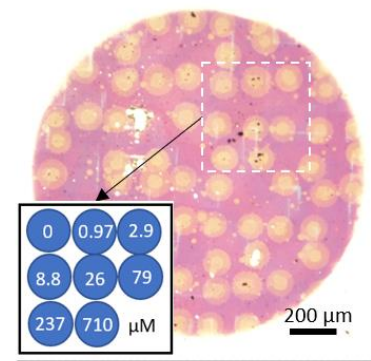

b)

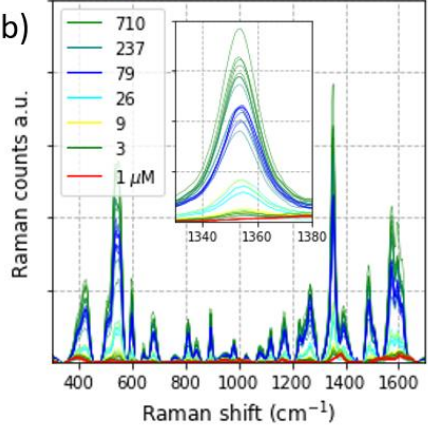

c)
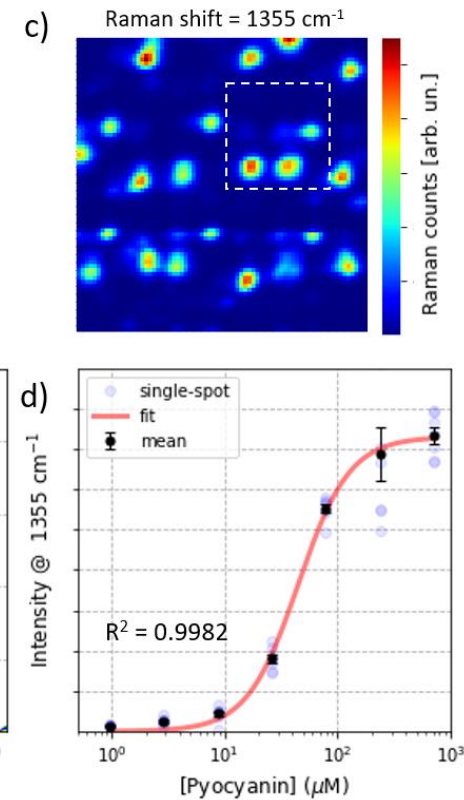

Figure S8. Microscope image of a sensor spotted with eight concentration of Pyocyanin $(0,0.97,2.9,8.8,26,79,237$ and 710 $\mu M)$ (a). SERS intensity map at the $1355 \mathrm{~cm}^{-1}$ Raman shift, which has been assigned to ring stretching vibrational modes [24]. (b). Spectra for each droplet in the map color coded by concentration (c). Raw and average intensity as a function of concentration. Fitting the averaged data to the Hill equation yields an $R^{2}$ of 0.9925 , with $K_{d}=4.80 \cdot 10^{-9} \mathrm{M}$ and $n=1.91$. 
Fig.S9. Quantitative analysis, repeatability

a1)

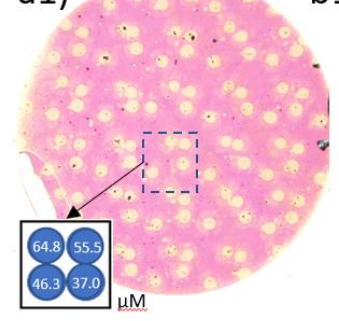

a2)

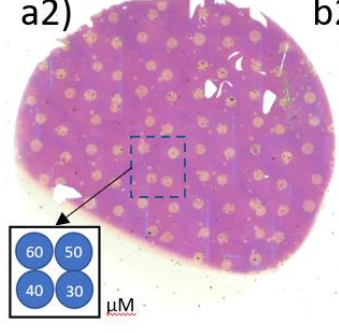

b1)

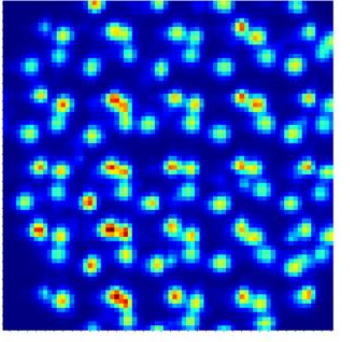

b2)

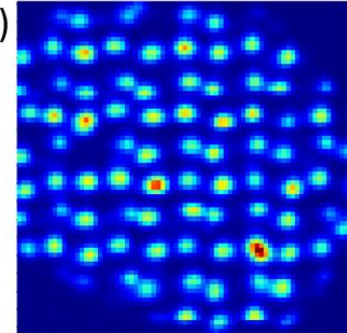

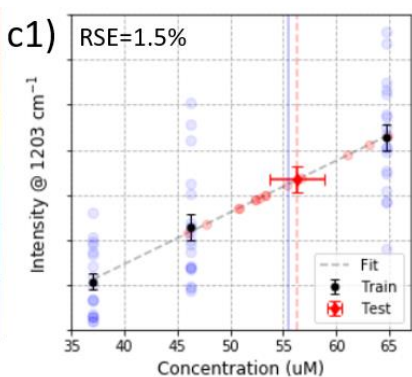

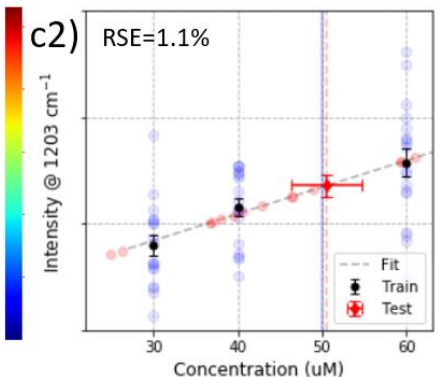

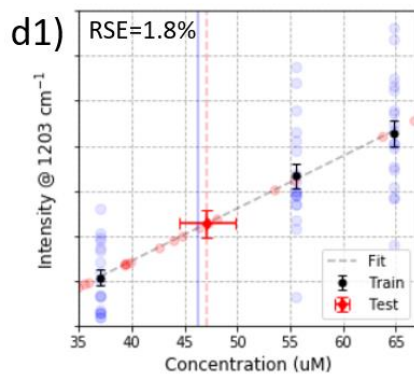

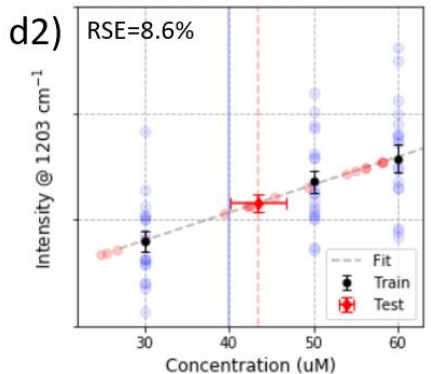

Figure S9. Microscope images (a1, a2) of two sensors spotted with four concentrations of BPE (reported in inset sketch). SERS intensity maps at $1203 \mathrm{~cm}^{-1}$ (b1, b2). Quantitation results on third highest (c1, c2) and second highest (d1, d2) concentration with model trained on remaining three concentrations. RSE for each measurement compared to the nominal concentration value reported in \% on figure.

Fig.S10. Effect of firing frequency and volume on drying time:

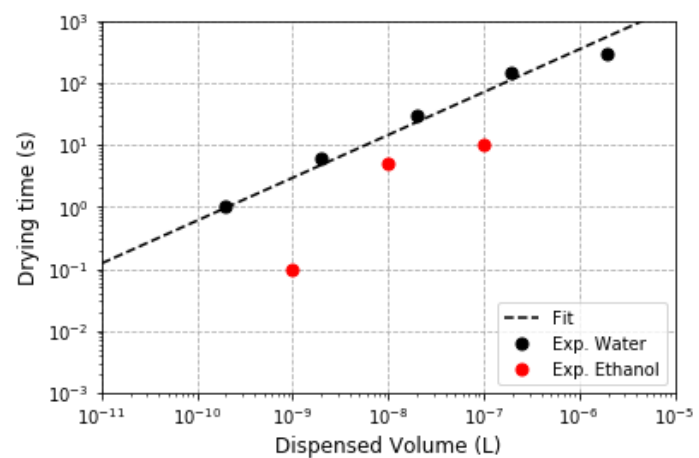

Figure S2. Drying time for inkjet dispensed droplets of different volumes (minimum volume $100 \mathrm{pL}$ ) for both water and ethanol (for comparison), measured with a video capable contact angle setup integrated in the printer. Linear fit (dotted) to extrapolate expected drying time for the single droplet (about $200 \mathrm{~ms}$ ).

\section{Appendix S1}

Here we prove the relation used in Supplementary Section S2 on "2D-Autocorrelation analysis of SERS maps", which we remind is:

$$
<G_{\delta}>=\frac{<I>^{2}}{<I^{2}>}
$$

This relation can be proved in the 1D continuous case for simplicity, by noting that: 


$$
<G_{\delta}>=\frac{\int_{0}^{D} G(\delta) d \delta}{D}=\frac{1}{D} \int_{0}^{D} \frac{1}{D<I^{2}>} \int_{0}^{D} I(x-\delta) I(x) d x d \delta
$$

Where $D$ is the span of the signal in the 1-dimentional case (replacing $N^{2}$ in the 2D case). Now one can implement a change of variable $\bar{x}=x-\delta$, to obtain:

$$
\begin{gathered}
\frac{1}{D^{2}<I^{2}>} \int_{0}^{D} \int_{x}^{x-D} I(\bar{x}) I(x) d x(-d \bar{x}) \\
=-\frac{1}{D^{2}<I^{2}>} \int_{0}^{D} I(x)\left[\int_{x}^{x-D} I(\bar{x}) d \bar{x}\right] d x \\
=\frac{1}{D^{2}<I^{2}>} \int_{0}^{D} I(x) D<I>d x \\
=\frac{D^{2}<I>^{2}}{D^{2}<I^{2}>}
\end{gathered}
$$

Which proves the relation above. The concept can be extended to 2-dimensions and the discrete case in a similar fashion.

\section{Bibliography}

[1] W. H. K. H. S. L. K. H. P. S. J. S. Jong Uk Lee, "Quantitative and Specific Detection of Exosomal miRNAs for Accurate Diagnosis of Breast Cancer Using a Surface-Enhanced Raman Scattering Sensor Based on Plasmonic Head-Flocked Gold Nanopillars," Small, p. 1804968, 2019.

[2] S.-G. P. J. K. X. X. V. G. S. A. M. D.-H. K. J. C. Xiaokun Wang, "Sensitive and Reproducible Immunoassay of Multiple Mycotoxins Using Surface-Enhanced Raman Scattering Mapping on 3D Plasmonic Nanopillar Arrays," Small, vol. 14, p. 1801623, 2018.

[3] L. M. J. L. Z. X. D. Z. Y. L. Z. Z. Sumeng Zou, "Quantification of trace chemicals in unknown complex systems by SERS," Talanta, vol. 186, pp. 452-458, 2018.

[4] S.-X. L. Z.-P. C. Y. C. R.-Q. Y. Xue-Qin Zhang, "Quantitative SERS analysis based on multiple-internalstandard embedded core-shell nanoparticles and spectral shape deformation quantitative theory," Chemometrics and Intelligent Laboratory Systems, vol. 177, pp. 47-54, 2018.

[5] T. R. M. S. S. A. H. T. Kaiyu Wu, "Optimizing silver-capped silicon nanopillars to simultaneously realize macroscopic, practical-level SERS signal reproducibility and high enhancement at low costs," Journal of Raman Spectroscopy, p. 1-11, 2017. 
[6] Z.-P. C. Y. C. Q. L. R.-Q. Y. Cai-Xia Shi, "Quantification of dopamine in biological samples by surfaceenhanced Raman spectroscopy: Comparison of different calibration models," Chemometrics and Intelligent Laboratory Systems, vol. 169, pp. 87-93, 2017.

[7] A. T. E. D. E. S. D. C. A. W. F. I. J. A. Guicheteau, "Reassessing SERS enhancement factors: using thermodynamics to drive substrate design," Faraday Discussions, vol. 205, p. 547, 2017.

[8] P. W. Q. L. X. W. X. H. Yunfei Tian, "Mapping for total surface-enhanced Raman scattering to improve its quantification analysis," Talanta, vol. 161, pp. 151-156, 2016.

[9] G. M. P. Z. Q. H. Z. L. X. H. X. W. Z. H. F. L. N. W. Chuhong Zhu, "A Hierarchically Ordered Array of Silver-Nanorod Bundles for Surface-Enhanced Raman Scattering Detection of Phenolic Pollutants," Advanced Materials, vol. 28, p. 4871-4876, 2016.

[10] L. C. Z. S. D. D. Y. C. Y. X. S. W. X. L. Y. Z. Y. S. Z. C. W. T. Yuxiu Zou, "Stable and unique graphitic Raman internal standard nanocapsules for surface-enhanced Raman spectroscopy quantitative analysis," Nano Research, vol. 9, no. 5, p. 1418-1425, 2016.

[11] W. E. H. L. C. M. W. Yun Wang, "Single cell stable isotope probing in microbiology using Raman microspectroscopy," Current Opinion in Biotechnology, vol. 41, pp. 34-42, 2016.

[12] X. L. C. J. C. L. H. L. J. H. S. W. G. L. X. Y. Q. Z. B. R. Wei Shen, "Reliable Quantitative SERS Analysis Facilitated by Core-Shell Nanoparticles with Embedded Internal Standards," Angew.andte Zuschriften, vol. 127, p. 7416-7420, 2015.

[13] T. V.-D. Andrew M. Fales, "Silver embedded nanostars for SERS with internal reference (SENSIR)," Journal of Material Chemistry C, vol. 3, no. 28, pp. 7319-7324, 2015.

[14] Z.-P. C. J.-W. J. Y. C. R.-Q. Y. Jing Song, "Quantitative surface-enhanced Raman spectroscopy based on the combination of magnetic nanoparticles with an advanced chemometric model," Chemometrics and Intelligent Laboratory Systems, vol. 135, pp. 31-36, 2014.

[15] Z.-P. C. Y. C. J.-W. J. R.-Q. Y. Tian-Hong Xia, "Improving the quantitative accuracy of surfaceenhanced Raman spectroscopy by the combination of microfluidics with a multiplicative effects model," Analytical Methods, vol. 6, p. 2363, 2014.

[16] M. P. F. G. B. T. R. T. S. A. M. S. S. A. B. J. J. a. Q. L. Jaeyoung Yang, "Surface-Enhanced Raman Spectroscopy Based Quantitative Bioassay on Aptamer-Functionalized Nanopillars Using LargeArea Raman Mapping," ACS Nano, vol. 7, no. 6, pp. 5350-5359, 2013.

[17] M. Y. Y. M. W. J. J. Z. Xiaohong Jiang, "Cysteamine-Modified Silver Nanoparticle Aggregates for Quantitative SERS Sensing of Pentachlorophenol with a Portable Raman Spectrometer," Applied Materials and Interfaces, vol. 5, no. 15, pp. 6902-6908, 2013. 
[18] K.-S. J. J.-H. H. H. K. S. K. Y. D. S. J.-M. N. Dong-Kwon Lim, "Highly uniform and reproducible surface-enhanced Raman scattering from DNA-tailorable nanoparticles with 1-nm interior gap," Nature Nanotechnology, vol. 6, p. 452-460, 2011.

[19] K. R. A. D. M. T. B. T. H. J. P. Anne Marz, "Towards a quantitative SERS approach - online monitoring of analytes in a microfluidic system with isotope-edited internal standards," Journal of Biophotonics, vol. 2, no. 4, p. 232-242, 2009.

[20] A. M. M. Sackmann, "Surface enhanced Raman scattering (SERS) - a quantitative analytical tool?†," Journal of Raman Spectroscopy, vol. 37, pp. 305-310, 2006.

[21] Z.-P. C. J.-W. J. R.-Q. Y. Yao Chen, "Quantitative determination of ametryn in river water using surface-enhanced Raman spectroscopy coupled with an advanced chemometric model," Chemometrics and Intelligent Laboratory Systems, vol. 142, p. 166-171, 2015.

[22] Private communication with Prof. Jaebum Choo, (Chung-Ang University, South Korea).

[23] Marco Pagliai, Stefano Caporali, Maurizio Muniz-Miranda, Giovanni Pratesi, and Vincenzo Schettino SERS, XPS, and DFT Study of Adenine Adsorption on Silver and Gold Surfaces, J. Phys. Chem. Lett. $2012,3,2,242-245$

[24] G. B., V. M.-G., V. L.-P., E. H., C. H., M. N. S.-O., S. R.-C., C. C., S. C., I. P.-J., L. S., A. L. P., J. P.-J., I. P.-S. and L. M. L-M. Detection and imaging of quorum sensing in Pseudomonas aeruginosa biofilm communities by surface-enhanced resonance Raman scattering. Nature Materials volume 15, 12031211 (2016) 